\title{
Spontaneous Symmetry Breaking in Interdependent Networked Game
}

SUBJECT AREAS:

COMPLEX NETWORKS

NONLINEAR PHENOMENA

COMPUTATIONAL MODELS

Received

21 October 2013

Accepted

23 January 2014

Published

14 February 2014

Correspondence and requests for materials should be addressed to L.W. (fdlwang@gmail. com); C.-Y.X.

(xialooking@163. com) or Z.W. (zhengwang0@gmail. com)

\author{
Qing Jin ${ }^{1,2}$, Lin Wang ${ }^{3}$, Cheng-Yi Xia ${ }^{4}$ \& Zhen Wang ${ }^{2,5,6}$
}

${ }^{1}$ Center for Complex Network Research and Department of Physics, Northeastern University, Boston, MA 02 115, USA, ${ }^{2}$ School of Physics, Nankai University, Tianjin 300071, China, ${ }^{3}$ Centre for Chaos and Complex Networks, Department of Electronic Engineering, City University of Hong Kong, Kowloon, Hong Kong, ${ }^{4}$ Key Laboratory of Computer Vision and System (Ministry of Education) and Tianjin Key Laboratory of Intelligence Computing and Novel Software Technology, Tianjin University of Technology, Tianjin 300384, China, ${ }^{5}$ Department of Physics, Hong Kong Baptist University, Kowloon Tong, Hong Kong, ${ }^{6}$ Center for Nonlinear Studies, Beijing-Hong Kong-Singapore Joint Center for Nonlinear and Complex systems (Hong Kong), and Institute of Computational and Theoretical Studies, Hong Kong Baptist University, Kowloon Tong, Hong Kong.

Spatial evolution game has traditionally assumed that players interact with direct neighbors on a single network, which is isolated and not influenced by other systems. However, this is not fully consistent with recent research identification that interactions between networks play a crucial rule for the outcome of evolutionary games taking place on them. In this work, we introduce the simple game model into the interdependent networks composed of two networks. By means of imitation dynamics, we display that when the interdependent factor $\alpha$ is smaller than a threshold value $\alpha_{C}$, the symmetry of cooperation can be guaranteed. Interestingly, as interdependent factor exceeds $\alpha_{C}$, spontaneous symmetry breaking of fraction of cooperators presents itself between different networks. With respect to the breakage of symmetry, it is induced by asynchronous expansion between heterogeneous strategy couples of both networks, which further enriches the content of spatial reciprocity. Moreover, our results can be well predicted by the strategy-couple pair approximation method.

$t$ is widely recognized that cooperation is a key force for the social and natural evolution ${ }^{1,2}$. To support this issue, social dilemma game plays a significant role in understanding the emergence of cooperation via introducing spatial structures ${ }^{3-5}$. However, most of these studies are implemented on a single network, which neglects the fact that other related systems also possess a certain influence on the evolution of cooperation, and vice versa ${ }^{6-29}$. Similar cases are ubiquitous in realistic life: for instance, when a global financial crisis breaks out, the future development of a country not only depends on its own stimulation policy, but also relies on the recovery status of other countries that build close economic connection with $\mathrm{it}^{30,31}$. In order to explore how cooperation evolves within different yet correlative systems, some more sophisticated paradigms that are close to realistic situations and can capture the interdependency between these systems need to be introduced ${ }^{32-34}$.

Recently, the study of interdependent networks, especially their property and function, becomes an active topic. One typical example is that Buldyrev et al. investigated the catastrophic cascade of failures on the interdependent networks, where the status of nodes on one network also lay on the nodes of other network ${ }^{35}$. They found that these networks were more vulnerable to random failures if they had broader degree distributions, which was contrary to the situation of one single network ${ }^{36}$. Inspired by these innovations, the model of interdependent networks becomes an appropriate candidate to explore the question about the evolution of cooperation within different yet correlative systems. However, how to construct interdependency between these networks seems immediately important ${ }^{37}$. Since the evolution of strategies is directly determined by individual fitness, the point-to-point interdependency (i.e., each node has a corresponding partner on the other network) through rescaling individual fitness becomes a potential approach. In this sense, a player's decision is not simply dependent on his own payoff during the game but also relevant to his companion's situation ${ }^{38-41}$. Looking at some examples more specifically, in a recent research paper ${ }^{42}$, where the biased utility function on interdependent networks was implemented, it was shown that the stronger the bias in the utility function, the higher the level of public cooperation. While in ${ }^{43}$, if individuals were allowed to engage in several layers of networks of interactions simultaneously, the multiplex structure enhancing the resilience of cooperative behaviors for extremely large values of the temptation was reported. In the line with these efforts, an interesting question poses itself, which we aim to address in what follows. Namely, if the fitness of individuals (who just engage in the interaction on their own network) is evaluated in a symmetric way, is this beneficial for the evolution of cooperation or not? 
In the present work, we will perform the Prisoner's Dilemma Game (PDG), one of the most powerful models in studying cooperation phenomenon, on the interdependent networks (which are composed of Network Up and Network Down, see Figure 1 for schematic representation). To characterize the mutual influence between two networks, an interdependent factor $\alpha(0 \leq \alpha \leq 1)$ is proposed: during the evaluation of fitness of focal individual, his own payoff occupies $\alpha$; while the remainder (namely, $1-\alpha$ ) comes from the payoff of his partner on another network, which is similar to the other-regarding trait ${ }^{44}$. Evidently, for $\alpha=0$ the most frequently adopted situation is recovered where two networks are completely independent. In the limit $\alpha \rightarrow 0$ two network are referred as weak interdependency; while in the limit $\alpha \rightarrow 1$ strong interdependency between networks will occur. We show that, when $\alpha$ is smaller than a threshold value $\alpha_{C}$, cooperation is highly promoted by setting a larger $\alpha$ and the fraction of cooperators on each network is symmetric (equal). On the contrary, if $\alpha$ exceeds $\alpha_{C}$, the spontaneous symmetry breaking between the fraction of cooperators on different networks can be observed. It is worth emphasizing that when the coupled-variable-replicator dynamics is allowed to regulate the evolution of different games in interdependent populations, the observation of spontaneous symmetry breaking also takes place ${ }^{39}$. In order to analyze and explain these phenomena, we also extend the traditional pair approximation and give out the strategy-couple pair approximation (SCPA, see the Supporting Information).

\section{Results}

We start by exploring the impact of interdependent factor $\alpha$ on the evolution of cooperation. Figure 2 shows the simulation and analysis results about how fraction of cooperators $\rho$ varies as a function of $b$ for different values of interdependent factor $\alpha$. To give a clear illustration, the value of $\rho$ is also provided when $b$ is smaller than 1 . As evidenced in the figure, we can observe two behaviors within the system: symmetry breaking phenomenon and phase transition. When $\alpha$ is smaller than a threshold value $\alpha_{C}\left(\alpha_{C} \approx 0.5\right.$ in the present model), the fraction of cooperators is symmetric on both networks (i.e., equal). At the same time, it is worth emphasizing that with increasing interdependent factor $\alpha$ cooperation can be better enhanced, which, to large extent, attributes to the self-organization of $C$ - $C$ coupled clusters, as we will discuss in what follows. However, when $\alpha$ exceeds $\alpha_{C}$ the spontaneous symmetry breaking will emerge, namely, fraction of cooperators on two networks is different, whereat its supporting condition is distinguished from the factor related with updating dynamics in previous literature ${ }^{39}$. In some particular regions (where $b$ is slightly larger than 1 ), all the players on one of the networks will uniformly choose the strategy $C$. With further

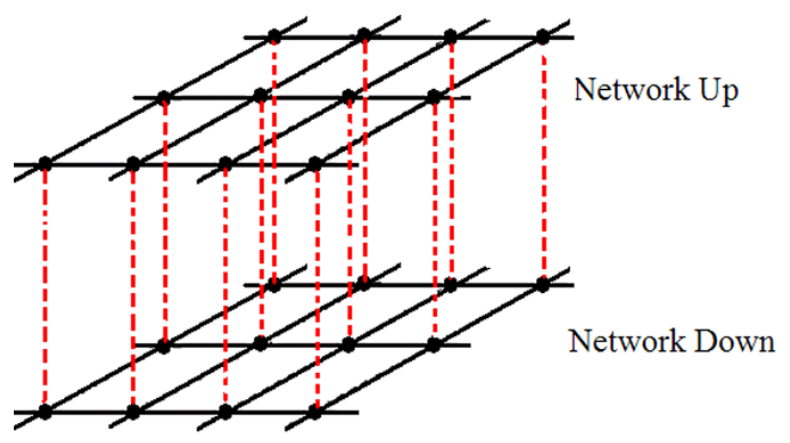

Figure $1 \mid$ Illustration of two interdependent networks, Network Up and Network Down. On each network, the nodes not only interact with their nearest neighbors, denoted by the black solid lines, but their situations also depend on the corresponding nodes on the other network, represented by the red dashed lines. Here, we only consider the case of one-one correspondence (i.e., point-to-point interdependency) between nodes on both networks. raising the temptation to defection, the symmetry of the system will be gradually regained. Moreover, Fig. 2(b) features the results of our SCPA approach (see the Supporting Information for more details), which can perfectly predict the enhancement of cooperation when the larger $\alpha$ is considered, and more importantly shows us the emergence of the spontaneous symmetry breaking phenomenon ${ }^{45-47}$. We restrict the value of $b$ between 0.95 and 1.10 in Fig. 2(b) to scrutinize the symmetry breaking phenomenon, since the pair approximation method is less reliable in dealing with the threshold value of cooperation (where the phase transition between mixed $C+D$ phase and pure $D$ phase occurs).

In order to study the phase transition in the system, now we turn to some typical cross sections of phase diagrams under different cases. Fig. 3(a) is the case of $\alpha=0.4$, which displays the existence of three sections: pure cooperators section (PC), mixed strategies section (MS) and pure defectors section (PD), which is resonant with previous report of PDG study ${ }^{23,28,47}$. However, in Fig. 3(b), where $\alpha$ is set as 0.9 (larger than $\alpha_{C}$ ), a novel section emerges: the symmetry breaking section (SB). In this section, usually with one of the networks showing a pure-cooperation behavior, two networks do not share the same fraction of cooperators. Notably, this interesting discovery (the coexistence of pure-cooperation and quasi-cooperation in both systems) can also be interpreted using the realistic instances. Take the maintenance of biological species as an example. Collective (pure) cooperation behavior is greatly beneficial for resisting the invasion of predators and further expanding the populations. On the other hand, individual survival is also faced with the temptation of obtaining high benefit yet no contribution, which thus leads to the existence of freeriders $^{1-3}$. We need to argue that if no additional rule is introduced, this pure-cooperation phenomenon goes beyond what can be supported by the traditional spatial reciprocity ${ }^{5}$. Moreover, it will be instructive to check the universality of this interesting behavior on other networks. From the presented results in Fig. 3(a) and Fig. 3(b), we find that the interdependent regular lattices and small-world (SW) networks actually share the same phase transition, implying that this behavior is robust to different coupled networks. It is worth mentioning that when the fraction of rewired links is very small, the transition details of cooperation behavior on the small-world network are identical with those of regular lattice. However, with the fraction of rewired links increasing, the extinction value of cooperators will also boost.

Importantly, the intriguing symmetry breaking phenomenon can also be obtained by applying the SCPA approach (see Fig. 3(c)). Instead of the second order phase transition in the simulation results (Fig. 3(b)), SCPA shows a first order phase transition, namely, it cannot provide the exact type of phase transition. The reason of this shortage is that SCPA only considers two couples' interaction within the system, while neglects the long-range interaction among players on the networks, which actually plays an important role in impacting the phase transition. This point is also good agreement with its prediction on traditionally single network, where the neglected role of loops in connectivity structure could cause more relevant deviation for most of networks (also see ${ }^{26}$ for more details). However, the flaw would not affect the prediction of SCPA about the existence of the symmetry breaking phenomenon. We also need to mention that there exists an unstable solution of the SCPA equations in the symmetry breaking phase (denoted by the dashed line), the details and equations of SCPA will be stated in the Supporting Information.

In order to explain the phenomenon of symmetry breaking, we subsequently proceed with examining the time evolution for four types of strategy couples: $C-C, C-D, D-C$ and $D-D$ couples. Figure 4 features the results obtained for $\alpha=0.4$ (a) and $\alpha=0.9$ (b), and the relevant evolution patterns are illustrated in Fig. 5. In the very early stages of evolution process (note that fractions are recorded in between full steps), $D$ - $D$ couples thrive. Quite surprisingly though, the tide changes fast, namely, $D-D$ couples become the 

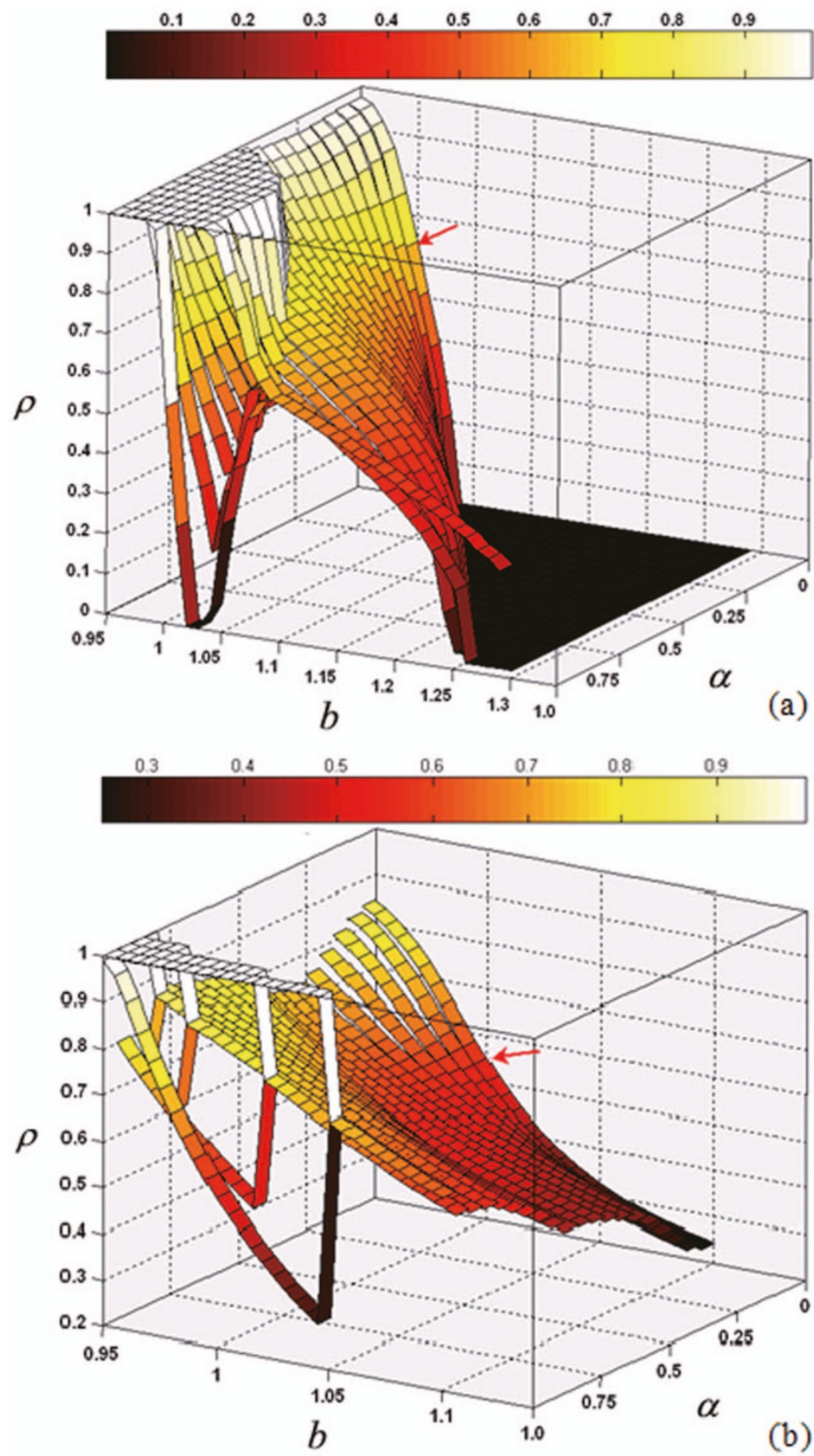

Figure $2 \mid$ Fraction of cooperators $\rho$ as a function $b$ for different values of $\alpha$ when Monte Carlo simulations (a) are implemented. Note that if $b$ is larger than 1 (marked by the red arrows), faction of cooperators will enhance with the increment of $\alpha$. When $\alpha$ exceeds a threshold value $\alpha_{C}\left(\alpha_{C} \approx 0.5\right)$, the symmetry breaking phenomenon will appear. In addition, our strategy-couple pair approximation approach (b) correctly predicts the trend (note that the range of both figures is different, since the analysis method is only for predicting the evolution trend).

rarest ones, and their dominant space is replaced by $C$ - $C$ couples. However, in the next thousands of steps, the situations will become different within two systems (see Fig. 4). For $\alpha=0.4$ the system will reach the equilibrium state very quickly with equal numbers of $D-C$ and $C$ - $D$ couples. For $\alpha=0.9$, however, the system needs a longer time to gain equilibrium, and at last only two types of couples (here $C-C$ and $D-C$ ) survive with the extinction of other couples (here $C-D$ and $D-D)$. In order to visually inspect this behavior, let us focus on the evolution patterns (see the bottom panel of Fig. 5). Initially, several sporadic clusters of $C-C, C-D$ and $D-C$ couples exist in the system, but soon they will combine to form larger clusters. Informed from the SCPA results (see Fig. 3(c)), we see that the present pattern is probably unstable, which means that until now the system merely reaches a metastable state and it can not survive from little perturbations. Interestingly, this prediction comes true in the next steps: two type of the clusters dies out at last and only two kinds of them survive, which results in the spontaneous symmetry breaking of the networks since the fractions of $C-D$ and $D-C$ are not equal any more. The $C-C$ 

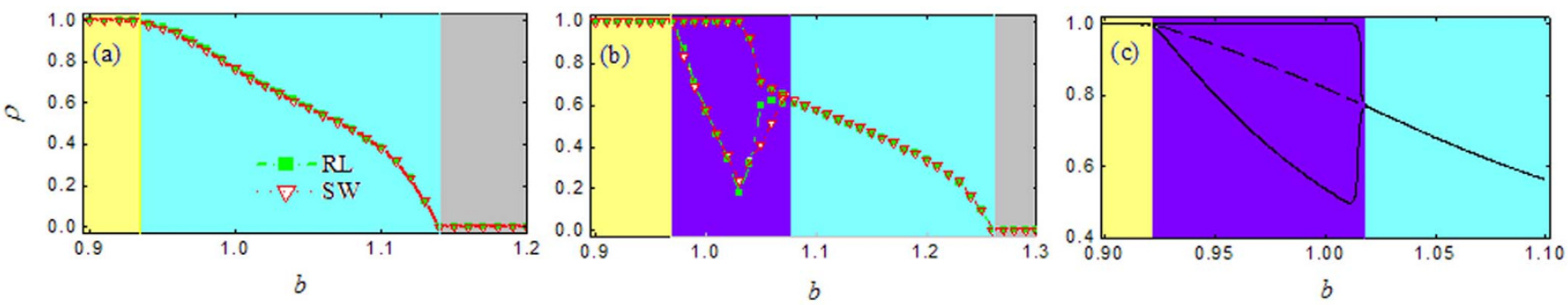

Figure 3 Typical cross sections of phase diagrams for $\alpha=0.4$ (a) and $\alpha=0.9$ (b) on the interdependent regular lattices (green squares) and small-world (SW) networks (red triangles) with fraction of rewired links equalling 0.05 . The colored regions represent different sections: yellow is pure cooperators section (PC), cyan is mixed strategies section (MS), gray is pure defectors section (PD) and purple is symmetry breaking section (SB). Additionally, the lines in (c) denote the results of strategy-couple pair approximation (SCPA) for $\alpha=0.9$, which is qualitatively similar to the case of (b). The dashed line is an unstable solution of SCPA approach (note that the range of figures is different, since the analysis method is only for predicting the evolution trend).

couples will remain in the system while the survival of $C-D$ or $D-C$ couples depends on the perturbations. Hence, we argue that when the interdependent networks can not strongly support the effective expansion of heterogeneous strategy couples on both networks, the breakage of symmetry becomes an inevitable outcome (namely, the asynchronous expansion between $C-D$ and $D-C$ couples is the hallmark signature indicating the emergence of spontaneous symmetry breaking).

Finally, it remains of interest to elucidate why cooperation can be improved with the increment of $\alpha$. To provide answers, we study the fraction distributions of strategy couples in Fig. 6. What firstly attracts our attention is the fact that the larger the value of $\alpha$ is, the more $C$ - $C$ couples exist. Actually, as increasing $\alpha$, there will be more $C-D, D-C$ and $D-D$ couples switching to $C-C$ couples (in the process, $D-D$ couples will first transform to $C-D$ or $D-C$ couples, then to $C-C$ couples). In addition, Fig. 5(c) shows the spatial patterns for different $\alpha$, whereby for $\alpha=0$ only a few sporadic $C$ - $C$ couple clusters exist, which come from the occasional superposition of cooperators' clusters on both networks, because the networks are non-relevant in such a situation. However, when a larger $\alpha$ is considered $(\alpha=0.2)$, more $C$ $C$ couples will be connected to each other in order to build solid clusters protecting themselves against the exploitation by defectors. When $\alpha$ equals to 0.4 (close to the symmetry breaking value $\alpha_{C}$ ), $C$ - $C$ couples strongly bond to each other, thereby much larger $C-C$ coupled clusters will be constructed in the system, which shows us a $C-C$ couples' ocean. At the same time, the $C-D$ and $D-C$ couples sporadically exist through forming small clusters and $D-D$ couples can only survive along the edges of these small mixture strategy coupled clusters.

\section{Discussion}

In conclusion, we have introduced the interdependent networks into spatial game study. Through systematic simulations, we have demonstrated that the interdependency between different networks has a great influence on the cooperative behavior. When the interdependent factor $\alpha$ exceeds a threshold value $\alpha_{C}$, the spontaneous symmetry breaking between the fraction of cooperators will appear, which can be regarded as a natural outcome of asynchronous expansion of heterogeneous strategy couples between networks. If it is smaller than $\alpha_{C}$, homogeneous fraction is able to be observed in the system and the fraction will increase with the increment of $\alpha$. Besides, these phenomena can be well predicted and analyzed by our strategy-couple pair approximation method.

Since the investigation of interdependent networks is a promising research topic, especially for the evolutionary games that can help to provide more comprehensive understanding of cooperative behavior, we hope that it will inspire further studies, such as, the effect of fitness asymmetry between entangled networks, coevolution of interdependent way and strategy updating. Moreover, it is also worth mentioning that the present model can be mapped onto a fourstrategy model (as reflected by the pair approximation method in
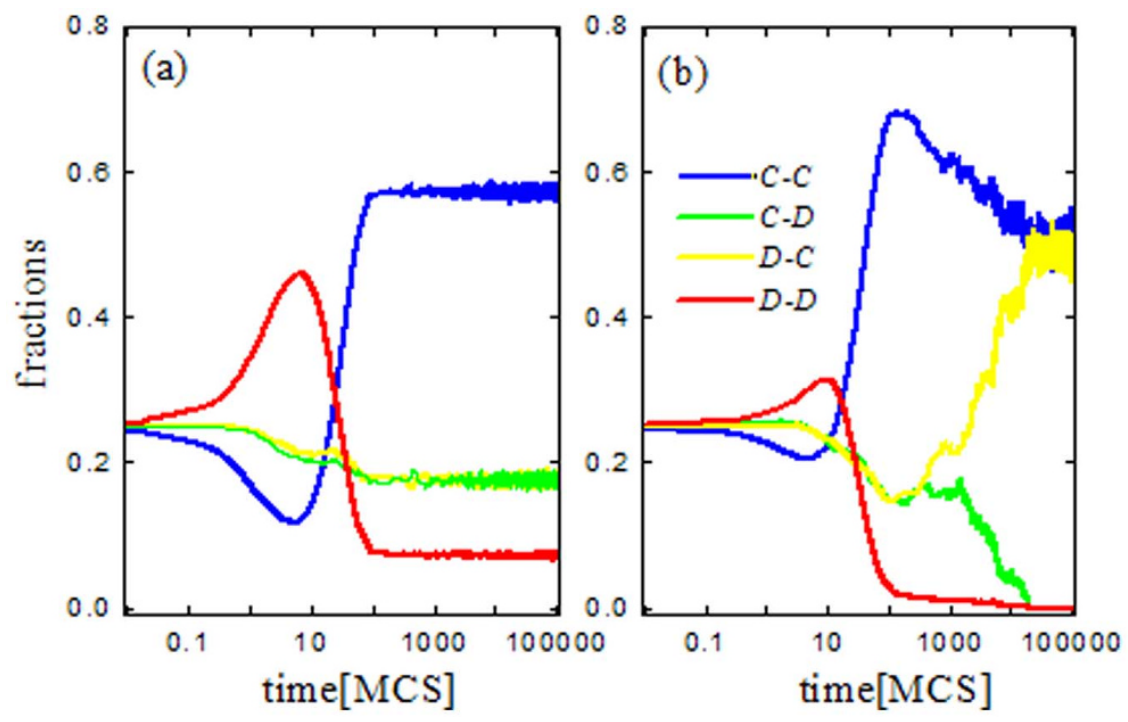

Figure $4 \mid$ Time evolution of different strategy couples for $\alpha=0.4$ (a) and $\alpha=0.9(\mathrm{~b})$. About the meanings of couples, we can give a simple example. For instance, $C$ - $D$ means that the player on Network Up chooses $C$ and the interdependent player on Network Down chooses $D$ (parameter: $b=1.005$ ). 

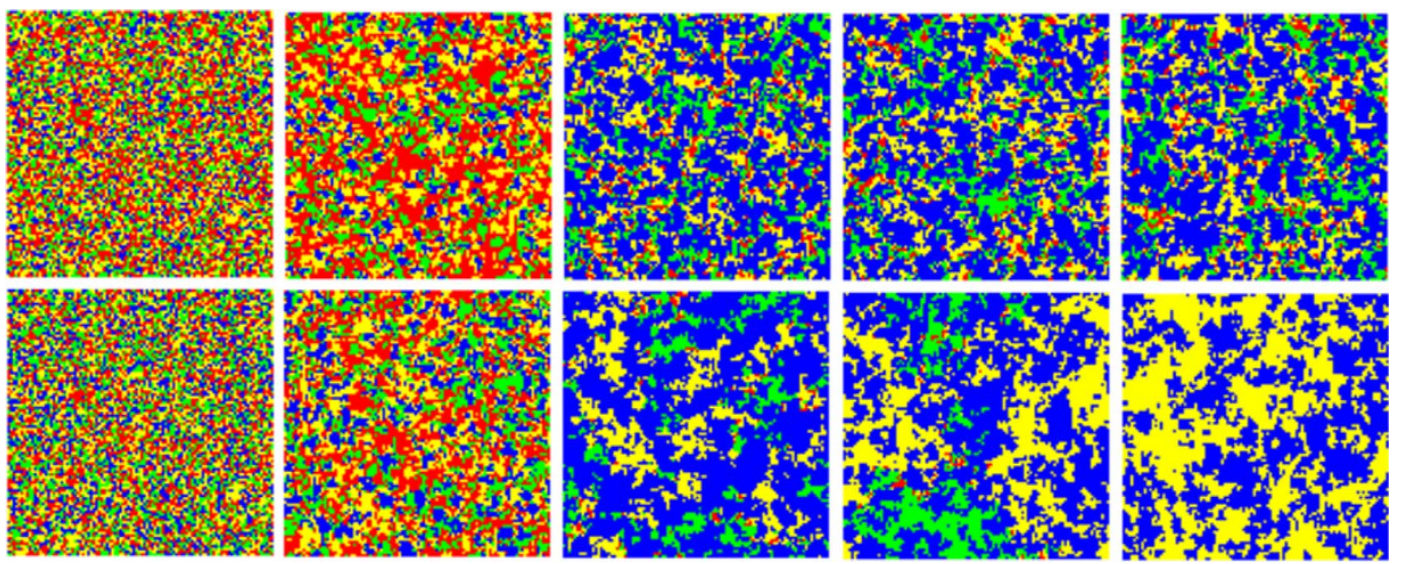

Figure $5 \mid$ Evolution patterns of different couples for $\alpha=\mathbf{0 . 4}$ (upper panel) and $\boldsymbol{\alpha}=\mathbf{0 . 9}$ (bottom panel). The color code of strategy couples is the same as Fig. 4, namely, $C-C$ blue, $C-D$ green, $D$ - $C$ yellow and $D-D$ red. From left to right the specific steps are $0,10,200,2000$, and 30000 for both panels (parameter: $b=1.005$ ).

Supporting Information) by a suitable dynamical rule, which will enrich the context of spatial reciprocity.

\section{Methods}

Evolutionary games on interdependent networks. As for the game, we will follow the Nowak-May framework, the so-called weak prisoner's dilemma game ${ }^{5}$. Two players have a choice between two pure strategies, cooperate $(C)$ and defect $(D)$. The payoffs are given by the following matrix:

$$
\begin{gathered}
C \\
C \\
C\left(\begin{array}{ll}
R & S \\
T & P
\end{array}\right)
\end{gathered}
$$

$R=1$ is the reward for mutual cooperation, $T=b$ temptation to defect, $S=0$ sucker's payoff and $P=0$ the punishment for mutual defection, whereby $1 \leq b \leq 2$ ensures a proper payoff ranking. In order to better estimate direct influences from the interdependency of networks, we choose the regular lattice where each node is connected to its four nearest neighbors and the small-world (SW) network with an average degree of four generated via the Watts-Strogatz algorithm ${ }^{48}$, since it is wellknown that heterogeneous networks, such as scale-free network ${ }^{49}$, would highly enhance cooperation in $\mathrm{PDG}^{8,17,18}$. Moreover, it is also worth mentioning that the interdependency between both networks is point-to-point, which means that every node in one network will have only one companion on the other network ${ }^{35}$.

The game is staged on two $L \times L$ square lattices (or small-world networks) with periodic boundary conditions. Each player $i$ is initially designated either as a cooperator or defector with equal probability and acquires the payoff by playing the game with all his neighbors (here player interacts with itself is not considered).
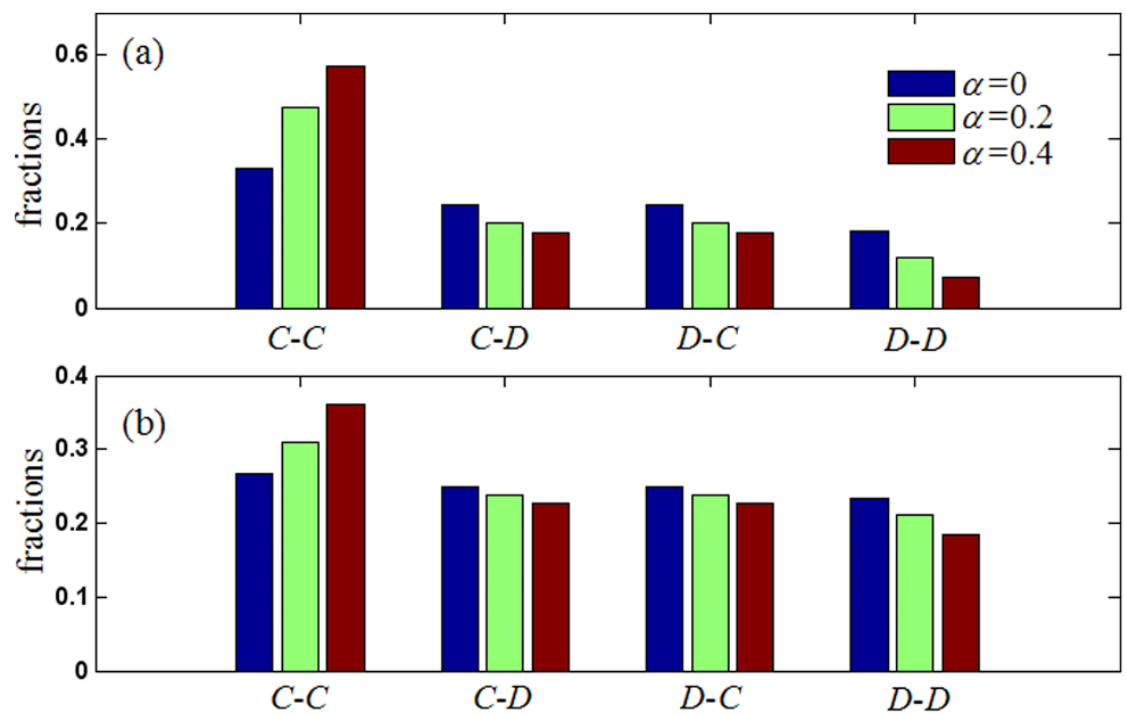

(c)

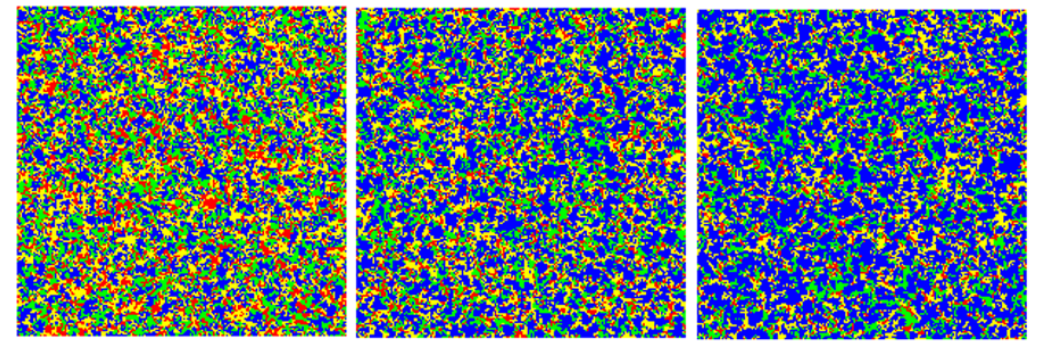

Figure 6 Fraction distributions of strategy couples for different value of $\alpha$ by using simulation (a) and SCPA approach (b). While (c) depicts the evolution patterns of stable status for different values of $\alpha$. From left to right $\alpha=0,0.2$ and 4.0, respectively. The color code of spatial patterns in (c) is the same as Fig. 4: $C$ - $C$ blue, $C$ - $D$ green, $D$ - $C$ yellow and $D-D$ red (parameter: $b=1.005$ ). 
Subsequently, player $i$ randomly selects one of his neighbors $j$ on the same network, and adopts his strategy based on the imitation dynamics ${ }^{50}$ (here the strategy invasion between different networks is not allowed):

$$
W(i \rightarrow j)=f\left(G_{j}-G_{i}\right)=\frac{1}{1+\exp \left[\left(-\left(G_{j}-G_{i}\right) / K\right)\right]},
$$

where $K$ represents the amplitude of noise (we simply fix $K$ to be 0.1 in this work), and $G_{i}$ denotes the fitness of player $i$, considering both his own payoff $P_{i}$ and the payoff of his partner $P_{i}^{\prime}$. Of particular interest, the fitness $G_{i}$ can be quantitatively evaluated in the following way $G_{i}=(1-\alpha) * P_{i}+\alpha * P_{i}^{\prime}$. Here $0 \leq \alpha \leq 1$ represents the interdependent factor. If $\alpha=0$, then the player's fitness is equal to his own payoff, and the model gets back to the original spatial PDG that have been extensively studied ${ }^{53-64}$. With the increment of $\alpha$ (i.e., $\alpha>0$ ), the fitness of one network depends on the status of another system. In particular, for $\alpha=1$, the fitness of a node will become fully determined by his companion's situation on the other network, and the strategies are adopted by means of a toss coin, which, herein, is distinguished from the research of voter model (that is closely related with topological features of networks) ${ }^{51,52}$. Notably, this model can be interpreted rather effectively. From the purely biological viewpoint, the survival and propagation of an agent is not only decided by his ability, but also depends on the quantity of his prey and predator. On other hand, especially in economic systems, the development of a company needs its own asset, but also involves the financial situation of his opponent and client.

Monte Carlo simulations results are obtained on the network with the size $L=100$ to 400 , yet when dealing with the phase transition points, a large size $(L=1000)$ is adopted to assure the accuracy of the simulation. In a full Monte Carlo step (MCS) each player has a chance to adopt the strategy from one of its neighbors once on average. Moreover, the key quantity fraction of cooperators $\rho$ is determined with the last $10^{4}$ full steps of overall $5 \times 10^{5} \mathrm{MCS}$, and the final data results from an average over 50 independent realizations.

1. Maynard-Smith, J. Evolution and the Theory of Games (Cambridge University Press, Cambridge, 1982).

2. Axelrod, R. The evolution of cooperation (Basic Books, New York, 1984).

3. Nowak, M. A. Five rules for the evolution of cooperation. Science 314, 1560-1563 (2006).

4. Hofbauer, J. \& Sigmund, K. Evolutionary Games and Population Dynamics (Cambridge University Press, Cambridge, UK, 1998).

5. Nowak, M. \& May, R. M. Evolutioanry games and spatial chaos. Nature 359 826-29 (1992)

6. Doebeli, M. \& Hauert, C. Models of cooperation based on prisoners dilemma and snowdrift game. Ecol. Lett. 8, 748-766 (2005).

7. Vainstein, M. H. \& Arenzon, J. J. Disordered environments in spatial games. Phys. Rev. E 64, 051905 (2001)

8. Abramson, G. \& Kuperman, M. Social games in a social network. Phys. Rev. E 63 , 030901(R) (2001).

9. Lozano, S., Arenas, A. \& Saánchez, A. Mezoscopic structure conditions the emergence of cooperation on social networks. PLoS ONE 3, e1892 (2008).

10. Wang, S., Szalay, M. S., Zhang, C. \& Csermely, P. Learning and innovative elements of strategy adoption rules expand cooperative network topologies. PLoS ONE 3, e1917 (2008)

11. Gracia-Lázaro, C., Cuesta, J., Sánchez, A. \& Moreno, Y. Human behavior in prisoners dilemma experiments suppresses network reciprocity. Sci. Rep. 2, 325 (2012).

12. Gómez-Gardeñes, J., Poncela, J., Floría, L. M. \& Moreno, Y. Natural selection of cooperation and degree hierarchy in heterogeneous populations. J. Theor. Biol. 253, 296-301 (2008)

13. Wu, B., Traulsen, A. \& Gokhale, S. C. Dynamic Properties of Evolutionary Multiplayer Games in Finite Populations. Games 4, 182-199 (2013).

14. Chen, X.-J., Perc, M. \& Szolnoki, A. Risk-driven migration and the collective-risk social dilemma. Phys. Rev. E 86, 036101 (2012).

15. Jiang, L.-L., Wang, W.-X., Lai, Y.-C. \& Wang, B.-H. Role of adaptive migration in promoting cooperation in spatial games. Phys. Rev. E 81, 036108 (2010).

16. Zimmermann, M. G., Eguíluz, V. \& Miguel, M. S. Coevolution of dynammical states and interactions in dynamics networks. Phys. Rev. E 69, 065102(R) (2004).

17. Santos, F. C. \& Pacheco, J. M. Scale-free networks provide a unifying framework for the emergence of cooperation. Phys. Rev. Lett. 95, 098104 (2005).

18. Santos, F. C., Pacheco, J. M. \& Lenaerts, T. Evolutionary dynamics of social dilemmas in structured heterogeneous populations. Proc. Natl. Acad. Sci. USA 103, 3490-3494 (2006)

19. Brede, M. Playing against the fittest: A simple strategy that promotes the emergence of cooperation. EPL 94, 30003 (2011).

20. Masuda, N. Participation costs dismiss the advantage of heterogenous networks in evolution of cooperation. Proc. R. Soc. B 274, 1815-1821 (2007).

21. Wu, Z.-X., Rong, Z.-H. \& Holme, P. Diversity of reproduction time scale promotes cooperation in spatial prisoners dilemma games. Phys Rev E 80, 036103 (2009).

22. Gómez-Gardeñes, J., Campillo, M., Floría, L. M. \& Moreno, Y. Dynamical organization of cooperation in complex topologies. Phys. Rev. Lett. 98, 108103 (2007).

23. Szabó, G., Vukov, J. \& Szolnoki, A. Phase diagrams for an evolutionary prisoner's dilemma game on two-dimensional lattices. Phys. Rev. E 72, 047107 (2005).
24. Tanimoto, J., Brede, M. \& Yamauchi, A. Network reciprocity by coexisting learning and teaching strategies. Phys. Rev. E 85, 032101 (2012).

25. Tanimoto, J. \& Sagara, H. Relationship between dilemma occurrence and the existence of a weakly dominant strategy in a two-player symmetric game. BioSystems 90, 105-114 (2007).

26. Szabó, G. \& Fáth, G. Evolutionary games on graphs. Phys. Rep. 447, 97-216 (2007).

27. Roca, C. P., Cuesta, J. \& Sánchez, A. Evolutionary game theory: temporal and spatial effects beyond replicator dyanmics. Phys. Life Rev. 6, 208-249 (2009).

28. Perc, M. \& Szolnoki, A. Coevolutionary games-a mini review. Biosystems 99, 109-125 (2010)

29. Perc, M., Gómez-Gardeñes, J., Szolnoki, A., Floría, L. M. \& Moreno, Y. Evolutionary dynamics of group interactions on structured populations: a review. J. R. Soc. Interface 10, 20120997 (2013).

30. Schweitzer, F. et al. Economic networks: The new challenges. Science 325, 422-425 (2009).

31. Huang, X., Havlin, I. V. S., \& Stanley, H. E. Cascading Failures in Bi-partite Graphs: Model for Systemic Risk Propagation. Sci. Rep. 3, 1219; DOI:10.1038/ srep01219 (2013)

32. Rybski, D., Buldyrev, S. V., Havlin, S., Liljeros, F. \& Makse, H. A. Communication activity in a social network: relation between long-term correlations and interevent clustering. Sci. Rep. 2, 560 (2012).

33. Mucha, P. J., Richardson, T., Macon, K., Porter, M. A. \& Onnela, J. P. Community structure in time-dependent, multiscale, and multiplex networks. Science $\mathbf{3 2 8}$ 876-878 (2010).

34. Barabási, A.-L. The network takeover. Nature Physics 8, 14-16 (2012).

35. Buldyrev, S. V., Parshani, R., Paul, G., Stanley, H. E. \& Havlin, S. Catastrophic cascade of failures in interdependent networks. Nature 464, 1025-1028 (2010).

36. Parshani, R., Paul, G., Stanley, H. E. \& Havlin, S. Interdependent networks: Reducing the coupling strength leads to a change from a first to second order percolation transition. Phys. Rev. Lett. 105, 048701 (2010).

37. Gao, J., Buldyrev, S. V., Stanley, H. E. \& Havlin, S. Networks formed from interdependent networks. Nature Physics 8, 40-48 (2012).

38. Tang, C.-B., Wang, Z. \& Li, X. Moderate intra-group bias maximizes cooperation on interdependent populations. PLoS ONE in press (2014)

39. Gómez-Gardeñes, J., Gracia-Lázaro, C., Floría, L. M. \& Moreno, Y. Evolutionary dynamics on interdependent populations. Phys. Rev. E 86, 056113 (2012).

40. Wang, Z., Szolnoki, A. \& Perc, M. Interdependent network reciprocity in evolutionary games. Sci. Rep. 3, 1183; DOI:10.1038/srep01183 (2013).

41. Wang, Z., Szolnoki, A. \& Perc, M. Optimal interdependence between networks for the evolution of cooperation. Sci. Rep. 3, 2470; DOI:10.1038/srep02470 (2013).

42. Wang, Z., Szolnoki, A. \& Perc, M. Evolution of pulbic cooperation on interdependent networks: The impact of biased utility functions. EPL 97, 48001 (2012).

43. Gómez-Gardeñes, J., Reinares, I., Arenas, A. \& Floría, L. M. Evolution of cooperation in multiplex networks. Sci. Rep. 2, 620 (2012).

44. Grund, T., Waloszek, C. \& Helbing, D. How Natural Selection Can Create Both Self- and Other-Regarding Preferences, and Networked Mind. Sci. Rep. 3, 1480 (2013).

45. González-Viñas, W. \& Arecchi, F. T. Order Parameter Fragmentation after a Symmetry-Breaking Transition. Phys. Rev. Lett. 83, 5210 (1999).

46. Ohtsuki, H., Nowak, M. A. \& Pacheco, J. M. Breaking the Symmetry between Interaction and Replacement in Evolutionary Dynamics on Graphs. Phys. Rev. Lett. 98, 108106 (2007)

47. Szabó, G., Szolnoki, A., Varga, M. \& Hanusovszky, L. Ordering in spatial evolutionary games for pairwise collective strategy updates. Phys. Rev. E 82, 026110 (2010)

48. Watts, D.-J. \& Strogatz, S. H. Collective dynamics of 'small-world' networks. Nature 393, 440-442 (1998).

49. Barabási, A. L. \& Albert, R. Emergence of scaling in random networks. Science $\mathbf{2 8 6}$ 509-512 (1999)

50. Szabó, G. \& Töke, C. Evolutioanry prisoner's dilemma game on a lattice. Phys. Rev. E 58, 69-73 (1998)

51. Suchecki, K., Eguíluz, V. M. \& San Miguel, M. Voter model dynamics in complex networks: Role of dimensionality, disorder, and degree distribution. Phys. Rev. E 72, 036132 (2005).

52. Sood, V. \& Redner, S. Voter Model on Heterogeneous Graphs. Phys. Rev. Lett. 94, 178701 (2005).

53. Zschaler, G., Traulsen, A. \& Gross, T. A Homoclinic Route to Full Cooperation in Adaptive Social Networks. New J. Phys. 12, 093015 (2010).

54. Fu, F., Wang, L., Nowak, M. A. \& Hauert, C. Evolutionary dynamics on graphs: Efficient method for weak selection. Phys. Rev. E 79, 046707 (2009).

55. Helbing, D. \& Yu, W. The outbreak of cooperation among success-driven individuals under noisy conditions. Proc. Natl. Acad. Sci. USA 106, 3680-3683 (2009).

56. Perc, M. \& Szolnoki, A. Social diversity and promotion of cooperation in the spatial prisoner's dilemma game. Phys. Rev. E 77, 011904 (2008).

57. Tanimoto, J., Brede, M. \& Yamauchi, A. Network reciprocity by coexisting learning and teaching strategies. Phys. Rev. E 85, 032101 (2012).

58. Dai, Q., Li, H., Cheng, H., Li, Y. \& Yang, J. Double-dealing behavior potentially promotes cooperation in evolutionary prisoner's dilemma games. New J. Phys. 12 113015 (2015). 
59. Szlnoki, A. \& Perc, M. Evolutioanry advantages of adaptive rewarding. New J. Phys. 14, 093016 (2012).

60. Poncela, J., Gómez-Gardeñes, J., Traulsen, A. \& Moreno, Y. Evolutionary game dyanmics in a growing structured population. New J. Phys. 11, 083031 (2009).

61. Ichinose, G., Saito, M. \& Suzuki, S. Collective chasing behavior between cooperators and defectors in the spatial prisoner's dilemma. PLoS ONE 8, e67702 (2013).

62. Meloni, S., Buscarino, A., Fortuna, L., Frasca, M., Gómez-Gardeñes, J., Latora, V. \& Moreno, Y. Effects of mobility in a population of prisoner's dilemma players. Phys. Rev. E 79, 067101 (2009).

63. Shigaki, K., Tanimoto, J., Wang, Z., Kokubo, S., Hgishima, A. \& Ikegaya, N. Referring to the social performance promotes cooperation in spatial prisoner's dilemma games. Phys. Rev. E 86,031141 (2012).

64. Ichinose, G., Saito, M., Sayama, H. \& Wilson, D. S. Adaptive long-range migration promotes cooperation under tempting conditions. Sci. Rep. 3, 2509 (2013)

\section{Acknowledgments}

This work has been partially supported by the National Natural Science Foundation of China (Grant No. 61374169).

\section{Author contributions}

Q.J., L.W., C.Y.X. and Z.W. designed framework; Q.J., L.W., Z.W. performed the research; Z.W. wrote the paper and supervised the whole process.

\section{Additional information}

Supplementary information accompanies this paper at http://www.nature.com/ scientificreports

Competing financial interests: The authors declare no competing financial interests.

How to cite this article: Jin, Q., Wang, L., Xia, C.-Y. \& Wang, Z. Spontaneous Symmetry Breaking in Interdependent Networked Game. Sci. Rep. 4, 4095; DOI:10.1038/srep04095 (2014).

(c) (i) () $\Theta$ This work is licensed under a Creative Commons AttributionNonCommercial-NoDerivs 3.0 Unported license. To view a copy of this license, visit http://creativecommons.org/licenses/by-nc-nd/3.0 